\title{
Evaluación del SAME-TT2R2 score en la calidad de la anticoagulación con warfarina
}

\section{Evaluation of SAME-TT2R2 score on the quality of anticoagulation with warfarin}

\author{
Carmenza Liliana Sandoval-Riveros, Fabio Alexánder Sierra-Matamoros, \\ Guillermo Mora-Pabón, Martha Rocío Hernández-Flechas, Jorge Alberto Vargas-Castro \\ - Bogotá, D.C. (Colombia)
}

\section{Resumen}

Introducción: la fibrilación auricular (FA) es la arritmia cardiaca más frecuente y está relacionada con eventos embólicos. La eficacia y seguridad de la warfarina en prevención del ictus depende del tiempo en rango terapéutico (TTR). Se han diseñado escalas para predecir el comportamiento del TTR al inicio de la terapia. El objetivo de este trabajo fue evaluar la capacidad predictiva y discriminativa del SAMeTT2R2 score en una población no caucásica.

Material y métodos: estudio analítico de cohorte retrospectiva en pacientes con FA no valvular tratados con warfarina entre 2013 y 2016 en una clínica de anticoagulación. Se caracterizó la muestra sociodemográfica y clínicamente. Se definió mal control de la anticoagulación como (TTR/ PINNR $<65 \%$ ). Se comparó el TTR/PINNR $<65 \%$ y SAMeTT2R $2<=2$ versus $>2$ con la prueba $\mathrm{U}$ de Mann-Whitney y chi cuadrado. Se construyeron curvas ROC para determinar discriminación de la escala. Se evaluó cada uno de sus elementos a través de regresión logística.

Resultados: se incluyeron 646 pacientes (edad media 77 años $\pm 8,8$ años, 51,4\% hombres). El TTR promedio fue $67.7 \%$ y el PINNR $63.6 \%$. Al comparar la frecuencia de pobre control entre SAMeTT2R2 score $\leq 2$ versus $>2$ con una prueba chi cuadrado, el PINNR fue significativo $(\mathrm{p}=0.004)$. La capacidad discriminativa de la escala para el TTR/PINNR fue baja (AUC: 0.52 y 0.54 respectivamente). No se encontró relación entre cada una de las variables que integran el SAMeTT2R2 score y el TTR/PINNR.

Conclusiones: la puntuación SAMeTT2R2 no mostró buena capacidad predictiva y discriminativa en la población estudiada. La calidad de la anticoagulación es el resultado de un proceso dinámico en relación con el cumplimiento, estado de salud o enfermedad intercurrente. (Acta Med Colomb 2019; 44: 75-81).

Palabras clave: SAMe-TT2R2 score, fibrilación auricular, anticoagulación, warfarina, tiempo en rango terapéutico.

\footnotetext{
Abstract

Introduction: atrial fibrillation (AF) is the most common cardiac arrhythmia and is related to embolic events. The efficacy and safety of warfarin in stroke prevention depends on the time in therapeutic range (TTR). Scales have been designed to predict the behavior of TTR at the start of therapy. The objective of this work was to evaluate the predictive and discriminative capacity of the SAMeTT2R2 score in a non-Caucasian population.

Material and methods: retrospective cohort analytical study in patients with non-valvular AF treated with warfarin between 2013 and 2016 in an anticoagulation clinic. The sample was demodemographically and clinically characterized. Poor control of anticoagulation was defined as (TTR / PINNR <65\%). The TTR / PINNR $<65 \%$ and SAMeTT2R $<=2$ versus $>2$ were compared with the Mann-Whitney $U$ test and chi square. ROC curves were constructed to determine scale discrimination. Each of its elements was evaluated through logistic regression.

Results: 646 patients were included (mean age 77 years \pm 8.8 years, $51.4 \%$ men). The average TTR was $67.7 \%$ and the PINNR $63.6 \%$. When comparing the frequency of poor control between
}

Dra. Carmenza Liliana Sandoval-Riveros: Residente de Medicina Interna, Universidad Nacional de Colombia; Dr. Fabio Alexánder Sierra-Matamoros: Psicólogo, Magister en Epidemiologia Clínica; Dr. Guillermo MoraPabón: Cardiólogo y Electrofisiólogo, Docente de la Universidad Nacional de Colombia; Dra. Martha Rocío Hernández-Flechas: Internista, Clínica de Anticoagulación, Clínica Universitaria Colombia, Clínica Colsanitas; Dr. Jorge AlbertoVargas-Castro.: Médico general, SURA EPS. Bogotá, D.C. (Colombia).

Correspondencia: Dra. Carmenza Sandoval, Bogota, D.C. (Colombia).

E-mail: calisari87@gmail.com

Recibido: 22/IX/2017 Aceptado: 22/III/2019 
SAMeTT2R 2 score $\leq 2$ versus $>2$ with a chi square test, the PINNR was significant $(p=0.004)$. The discriminative capacity of the scale for the TTR / PINNR was low (AUC: 0.52 and 0.54 respectively). No relationship was found between each of the variables that constitute the SAMeTT2R2 score and the TTR / PINNR.

Conclusions: the SAMeTT2R2 score did not show good predictive and discriminative capacity in the studied population. The quality of anticoagulation is the result of a dynamic process in relation to compliance, health status or intercurrent disease. Acta Med Colomb 2019; 44: 75-81).

Key words: $S A M e-T T 2 R 2$ score, atrial fibrillation, anticoagulation, warfarin, time in therapeutic range.

\section{Introducción}

La fibrilación auricular (FA) es la arritmia cardiaca más frecuente, aumentando su prevalencia con el incremento en la edad. Aproximadamente $12 \%$ de los pacientes con FA tienen 75-84 años de edad (1) y genera cinco veces mayor riesgo de accidente cerebrovascular, tres veces insuficiencia cardiaca y dos veces demencia, así como aumento en la mortalidad, alteraciones hemodinámicas, hospitalizaciones frecuentes y otros eventos tromboembólicos $(1,2)$. Los antagonistas de la vitamina $\mathrm{K}(\mathrm{AVK})$ han sido usados desde 1950 como anticoagulantes orales para la prevención del ictus en pacientes con FA; reducen el riesgo de accidente cerebrovascular isquémico en $64 \%$ con un número necesario a tratar de 37 pacientes durante un año para prevenir un primer accidente cerebrovascular (ACV), 12 con ACV o accidentes isquémicos transitorios (AIT) previos y disminuye la mortalidad por cualquier causa hasta un $26 \%$ en comparación con placebo (3). En la última década los inhibidores directos de la trombina y los inhibidores del factor Xa (anticoagulantes orales directos-ACOD) se han usado como alternativas a la warfarina porque no requieren monitoreo estrecho, su efecto terapéutico es predecible y son equiparables a la eficacia de la warfarina con menores tasas de sangrado (4).

Las guías internacionales $(1,5-7)$ recomiendan evaluar la calidad de la anticoagulación con cumarínicos a través del tiempo en rango terapéutico que ha mostrado una fuerte correlación con las tasas de sangrado y eventos tromboembólicos (8). Los métodos más usados para medirlo son el tiempo en rango terapéutico (TTR) y el porcentaje de tiempo en rango terapéutico (PINNR) que no son absolutamente intercambiables (9-12). Se acepta un TTR $>65 \%$ como objetivo en la terapia anticoagulante $(13,14)$. Cuando es menor a $40 \%$ implica un mal control e incrementa los riesgos tromboembólicos y hemorrágicos $(15,16)$. Sin embargo en la vida real esta meta es poco cumplida $(4,17,18)$ y su uso se limita por potenciales interacciones con alimentos, medicamentos y control estricto (19).

Apostolakis (20) publicó el puntaje SAMe-TT2R2 encontrando correlación de manera inversamente proporcional con el tiempo en rango terapéutico (TTR) con un punto de corte $>=2$ puntos. Sin embargo en validaciones externas hay hallazgos contradictorios en diferentes poblaciones $(9,21-$ 25). El objetivo del presente estudio fue evaluar la capacidad predictiva y discriminativa del SAMeTT2R2 score en la calidad de la anticoagulación con warfarina (medida por el TTR/PINNR) en los pacientes con fibrilación auricular no valvular quienes asistieron a una clínica de anticoagulación.

\section{Material y métodos}

La presente investigación es un estudio analítico de cohorte retrospectiva de pacientes con fibrilación auricular no valvular tratados con warfarina quienes asistieron a una clínica de anticoagulación durante enero de 2013 a junio de 2016 en diferentes ciudades de Colombia. Se consideró como exposición la clasificación de los pacientes según el SAM-TT2R2 y como desenlace la calidad de la anticoagulación.

Se calculó una muestra de 578 participantes a partir de un error tipo I de 0.05 , poder del $80 \%$, con un área bajo la curva entre 0.7 y 0.57 que corresponden al valor obtenido en el estudio de Apostokalis (20) y a su intervalo de confianza inferior (C-statistic: 0.7; IC95\%: 0.57-0.82)). Para el análisis, incluimos a pacientes con una edad mínima de 18 años, de nacionalidad colombiana, con diagnóstico confirmado de fibrilación auricular no valvular anticoagulados con warfarina que asistieron a controles del programa de anticoagulación y que contaron con al menos ocho valores consecutivos de INR (con una diferencia entre cada INR no mayor a siete semanas). Se excluyó del estudio a todos los pacientes con prótesis valvular, cardiopatía reumática, cáncer activo, extranjeros y aquellos en quienes no fue posible obtener de manera completa todas las variables definidas en el estudio.

Los pacientes con puntuación del SAM-TT2R2 (Tabla 1) entre 0-2 se clasificaron como bajo riesgo de mala calidad en la anticoagulación con warfarina y tres o más, como alto riesgo de mala calidad en la anticoagulación con warfarina. Se definió mala calidad de la anticoagulación un TTR/PINNR $<65 \%$ y buena calidad TTR/ PINNR $\geq 65 \%$. Se realizó la caracterización demográfica y clínica de la población por medio de frecuencias, medias, desviaciones, medianas y rangos intercuartílicos según la característica. El TTR es el porcentaje de días con INRs entre 2 y 3; se calcula por el método de Rosendaal (26). Es una interpolación lineal para asignar un valor de INR a cada día entre dos valores sucesivos; después el porcentaje de tiempo durante el cual los valores de INR interpolados se encuentran entre 2 y 3 es 
Tabla 1. SAMe-TT2R2 score (30).

\begin{tabular}{|l|c|}
\hline Ítem a evaluar & Puntaje \\
\hline Sexo femenino & 1 \\
\hline Edad menor de 60 años de edad & 1 \\
\hline Antecedentes médicos: más de dos comorbilidades ${ }^{1}$ & 1 \\
\hline Interacción medicamentosa (amiodarona) & 1 \\
\hline Uso de tabaco en los dos últimos años & 2 \\
\hline Raza no caucásica & 2 \\
\hline $\begin{array}{l}{ }^{1} \text { Hipertensión arterial, diabetes mellitus, enfermedad coronaria o infarto del miocardio } \\
\text { previo, enfermedad vascular periférica, enfermedad cardiaca congestiva, accidente } \\
\text { cerebrovascular, enfermedad pulmonar y enfermedad hepática o renal. }\end{array}$ \\
\hline
\end{tabular}

calculado. El PINNR representa el porcentaje de visitas en rango terapéutico y se correlaciona bien con el TTR calculado por el método de Rosendaal $(r=0.99, \mathrm{p}<0.001)(9-12)$.

Se realizó la caracterización demográfica y clínica de la población por medio de frecuencias absolutas y relativas y medidas de tendencia central y dispersión. Para analizar la relación entre el SAMeTT2R2 y el TTR/PINNR se estimó la mediana del TTR/PINNR por cada punto del SAMeTT2R2; se comparó la frecuencia de pacientes con TTR/PINNR $<65 \%$ entre el grupo de pacientes con SAMeTT2R2 $=2$ versus $>2$ por medio de una prueba U de Mann-Whitney y de asociación $\mathrm{Ch}^{2}$. Para evaluar la utilidad del SAMeTT2R2 en predecir el control de la anticoagulación se construyeron curvas ROC a partir de los puntajes SAMeTT2R2 con el fin de valorar si otros puntos de corte son útiles para mejorar el valor predictivo de la escala; se calculó el C-statistic y la sensibilidad y especificidad de los puntajes obtenidos en el SAMeTT2R2.

Una vez determinada la capacidad del SAMeTT2R2 para predecir o no, el control de la anticoagulación, se evaluó de manera individual cada uno de sus elementos por regresión logística considerando como variables de desenlace el TTR y el PINNR $<65 \%$ y como variables predictoras los factores que conforman el puntaje SAMeTT2R2. Los análisis se realizaron en el programa estadístico Stata $14^{\circledR}$ y R, asumiendo un error tipo I de 0.05 . Este trabajo se norma bajo la declaración de Helsinki (27) y fue aprobado por parte del comité de ética institucional.

\section{Resultados}

En 11 ciudades de Colombia se realizaron 3867 consultas a pacientes anticoagulados con diferentes diagnósticos y esquemas terapéuticos durante el mes de junio de 2016. Eran adultos con fibrilación auricular 906, a quienes se les aplicó los criterios de inclusión y exclusión y finalmente se obtuvo un número de 646 participantes (Figura 1). Los pacientes fueron seguidos durante un periodo mínimo de nueve meses y un máximo de tres años.

La edad promedio de la muestra fue 77 años con una desviación estándar de 8.80, la edad mínima fue 37 años y la máxima 98 años. El índice de masa corporal (IMC)

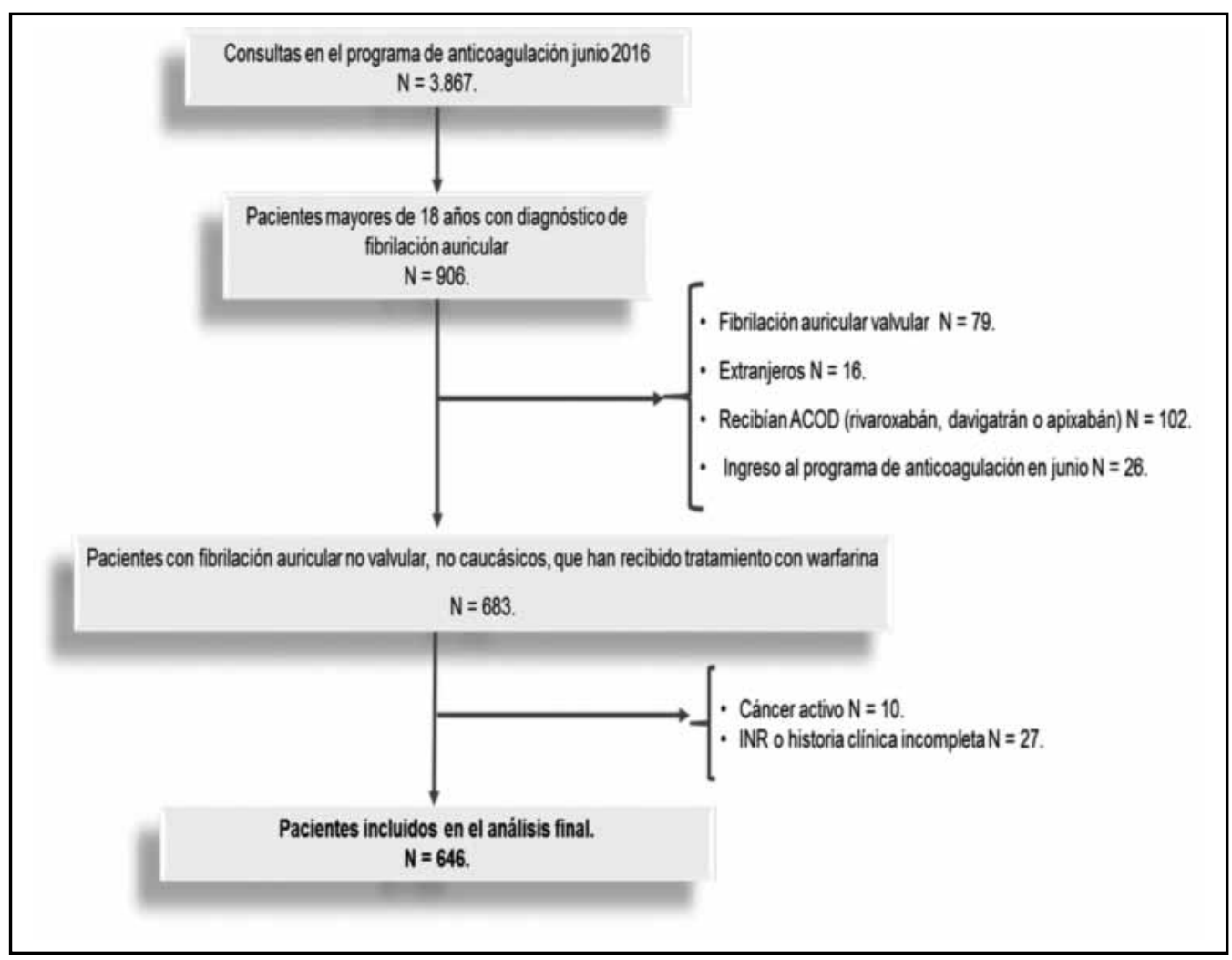

Figura 1. Diagrama de flujo del proceso de selección de la muestra. 
promedio fue 28.5. Dentro de las enfermedades mentales el diagnóstico más frecuente fue demencia tanto vascular como Alzheimer, seguido por depresión y ansiedad. El 70\% de la muestra tenía una puntuación CHA2DS2VASc $>=4$ puntos y $88.54 \%$ tenían un HAS-BLED Score $\leq 2$ puntos que representa un riesgo intermedio (probabilidad de sangrado en un año 1.88-3.2\%). La Tabla 2 muestra las principales características de la población.

Todos los pacientes por ser no caucásicos tenían una puntuación mínima de dos puntos que son los otorgados por tener esta característica, por lo que el punto de corte para correlación con mala calidad de la anticoagulación, fue mayor de dos puntos. El $75.23 \%$ de los participantes tenían una puntuación mayor de dos puntos. El TTR promedio calculado por el método de Rosendaal fue $67.7 \%$ con valores que oscilaron entre 0 y $100 \%$. El PINNR promedio

Tabla 2. Principales características de los pacientes incluidos.

\begin{tabular}{|c|c|c|}
\hline \multicolumn{2}{|l|}{ Característica } & Frecuencia $(\%)$ \\
\hline \multicolumn{2}{|l|}{ Sexo femenino } & $314(48.61)$ \\
\hline \multicolumn{2}{|l|}{ Edad - Media (DE) } & $77(8.80)$ \\
\hline \multicolumn{2}{|l|}{ Edad $<60$ años } & $20(3.1)$ \\
\hline \multicolumn{2}{|l|}{ Hipertensión arterial } & $561(86.84)$ \\
\hline \multicolumn{2}{|l|}{ Diabetes } & $168(26.05)$ \\
\hline \multicolumn{2}{|l|}{ IMC - Media (DE) } & $28,5(6.15)$ \\
\hline \multicolumn{2}{|l|}{$\mathrm{IMC} \geq 30 \mathrm{Kg} / \mathrm{m}^{2}$} & $148(22.95)$ \\
\hline \multicolumn{2}{|l|}{ Enfermedad hepática } & $4(0.62)$ \\
\hline \multicolumn{2}{|l|}{ Dislipidemia } & $500(77.40)$ \\
\hline \multicolumn{2}{|c|}{ Cardiopatía isquémica o enfermedad coronaria } & $151(23.37)$ \\
\hline \multicolumn{2}{|l|}{ ECV/AIT } & $114(17.65)$ \\
\hline \multicolumn{2}{|l|}{ Insuficiencia cardiaca } & $111(17.18)$ \\
\hline \multicolumn{2}{|c|}{ Enfermedad arterial periférica } & $29(4.49)$ \\
\hline \multicolumn{2}{|l|}{ EPOC } & $139(21.52)$ \\
\hline \multicolumn{2}{|l|}{ TFG $<30 \mathrm{~mL} / \mathrm{min}$} & $39(6.04)$ \\
\hline \multicolumn{2}{|l|}{ Diálisis } & $1(0.15)$ \\
\hline \multicolumn{2}{|l|}{ Tabaquismo } & $10(1.55)$ \\
\hline \multicolumn{2}{|c|}{ Tratamiento con amiodarona } & $156(24.15)$ \\
\hline \multicolumn{2}{|l|}{ Enfermedad mental } & $42(6.50)$ \\
\hline \multicolumn{2}{|l|}{ SAHOS } & $91(14.09)$ \\
\hline \multicolumn{2}{|c|}{ Cáncer tratado-en remisión } & $46(7.12)$ \\
\hline \multirow{10}{*}{ CHA2DS2VASc score } & 0 puntos & $2(0.31)$ \\
\hline & 1 punto & $16(2.48)$ \\
\hline & 2 puntos & $83(12.85)$ \\
\hline & 3 puntos & $146(22.60)$ \\
\hline & 4 puntos & $205(31.73)$ \\
\hline & 5 puntos & $97(15.02)$ \\
\hline & 6 puntos & $71(10.99)$ \\
\hline & 7 puntos & $22(3.41)$ \\
\hline & 8 puntos & $3(0.46)$ \\
\hline & 9 puntos & $1(0.15)$ \\
\hline & 0 puntos & $17(2.63)$ \\
\hline & 1 punto & $325(50.31)$ \\
\hline HAS-BLED score & 2 puntos & $230(35.60)$ \\
\hline & 3 puntos & $64(9.91)$ \\
\hline & 4 puntos & $10(1.55)$ \\
\hline $\begin{array}{l}\text { ECV/AIT: enfermedad } \\
\text { fermedad pulmonar ob } \\
\text { síndrome de apnealhipo }\end{array}$ & $\begin{array}{l}\text { accidente } \\
\text { TFG: tasc }\end{array}$ & $\begin{array}{l}\text { insitorio; } E P O C \text { : } \\
\text { glomerular; } S A L\end{array}$ \\
\hline
\end{tabular}

fue $63.6 \%$. El percentil 5 para el TTR fue $30.66 \%$ y para el PINNR fue $25 \%$. Más del $50 \%$ de los participantes tenían un control adecuado de anticoagulación (TTR/PINNR >65\%). Inicialmente se realizó un análisis descriptivo calculando la mediana del TTR/PINNR por cada una de las puntuaciones del SAMe-TT2R2 (Tabla 3). No se observó que a mayor puntuación del score, la mediana del TTR/PINNR disminuyera.

Al comparar las medianas del TTR/PINNR con el puntaje del SAMe-TT2R 2 estratificado en dos grupos, $\leq 2$ versus $>2$ puntos (Tabla 4) se encontró diferencia estadísticamente significativa cuando se empleó el método PINNR para cada uno de los grupos del score (U de Mann-Whitney=2.22, $\mathrm{p}=0.02$ ).

Se realizó una prueba chi cuadrado para comparar la frecuencia de pacientes con pobre control de la anticoagulación entre el grupo con SAMeTT2R2 score $\leq 2$ versus $>2$. Se halló diferencia significativa entre el grupo con un score menor o igual a 2, cuando se empleó el método PINNR (p 0.004) con mejor anticoagulación que no tuvo diferencia estadística con el TTR. Se observaron áreas bajo la curva cercanas a 0.5 (TTR=0.5285 PINNR=0.5487) indicando falta de discriminación del SAMeTT2R2 para predecir mal control de la anticoagulación (TTR/PINRR $<=65 \%$ ) (Figura 2).

$\mathrm{Al}$ analizar la curva ROC para un TTR/PINNR $<36 \%$ que fue el percentil 5 del estudio original, los resultados fueron 0,508 (IC95\% 0.428-0.588) y AUC 0.542 (IC95\% $0.458-0.625$ ) respectivamente. Dado a que el SAMeTT2R2 score no mostró una buena discriminación, ni tomando otros puntos de corte como referencia, se analizó cada una de las variables que lo conforman para determinar si estas predecían un pobre control de la anticoagulación y si esta capacidad se perdía al integrarlas al score. Ninguna de las variables se relacionó con el riesgo para una mala calidad de la anticoagulación con warfarina, lo cual puede explicar la pobre capacidad de discriminación del score.

Tabla 3. Mediana del TTR/PINNR por puntuación del SAMe-TT2R2 score.

\begin{tabular}{|c|c|c|c|}
\hline SAMe-TT2R2 score & N & $\begin{array}{c}\text { TTR } \\
\text { Mediana (RIQ) }\end{array}$ & $\begin{array}{c}\text { PINNR } \\
\text { Mediana (RIQ) }\end{array}$ \\
\hline 2 & 160 & $73.7(57.25-84.85)$ & $75(50-75)$ \\
\hline 3 & 286 & $68.9(55.2-80.9)$ & $62.5(50-75)$ \\
\hline 4 & 158 & $69.65(50.6-82.2)$ & $62.5(50-75)$ \\
\hline 5 & 37 & $67.7(48.9-84.3)$ & $62.5(37.5-75)$ \\
\hline 6 & 5 & $76.6(75.3-81.3)$ & $75(75-75)$ \\
\hline
\end{tabular}

Tabla 4. Mediana del TTR/PINNR de acuerdo al puntaje $<=a 2 o>$ del SAMe-TT2R2 score.

\begin{tabular}{|c|c|c|c|}
\hline SAMe-TT2R2 score & N & $\begin{array}{c}\text { TTR } \\
\text { Mediana (RIQ) }\end{array}$ & $\begin{array}{c}\text { PINNR } \\
\text { Mediana (RIQ) }\end{array}$ \\
\hline$<=2$ puntos & 160 & $73.7(57.25-84.85)$ & $75(50-75)$ \\
\hline$>2$ puntos & 486 & $69.35(53.4-81.9)$ & $62.5(50-75)$ \\
\hline
\end{tabular}




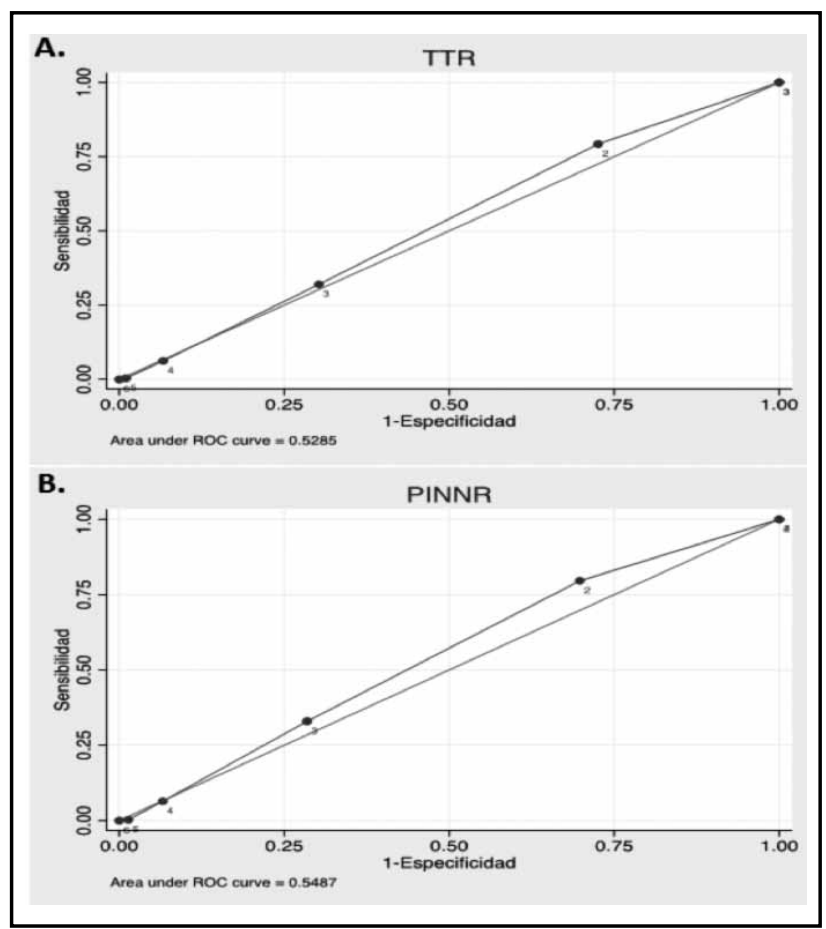

Figura 2. Capacidad discriminativa del SAMeTT2R2 score frente a la calidad de la anticoagulación. (A. De acuerdo con el TTR. B. Según el PINNR).

\section{Discusión}

En este estudio, se aplicó la puntuación SAME-TT2R2 a una población no caucásica de pacientes con FA no valvular seguidos en clínicas de anticoagulación de diferentes regiones de Colombia. El hallazgo más importante es que el score no es útil para discriminar pacientes que van a tener un mal control de la anticoagulación con warfarina determinado por el TTR o el PINNR, incluso con puntos de corte diferentes al planteado por Apostolakis. En la caracterización de la muestra no hubo diferencia significativa entre la frecuencia del sexo y la edad similar a lo encontrado en la literatura (28-30), excepto en el estudio de Apostolakis (20), en donde las mujeres fueron el $67.1 \%$ en la cohorte de validación externa y los menores de 60 años fueron 14.3\%, 14.4\% y $10.4 \%$ en la cohorte de derivación, de validación interna y externa respectivamente. El $73.84 \%$ de la muestra tuvo un nivel educativo bueno, un paciente con bajo nivel educativo puede presentar pobre nivel de interiorización sobre su enfermedad y tratamiento. Es una comorbilidad común la HTA y dislipidemia ( $87 \%$ y $77.4 \%$ respectivamente) en los diferentes artículos (28-30); en cuanto a obesidad y tabaquismo activo hubo variación con respecto a la muestra de validación externa que tuvo un mayor porcentaje con $43.2 \%$ y $49 \%$ respectivamente.

Varias investigaciones se han realizado para tratar de encontrar factores relacionados con la mala calidad en la anticoagulación con el fin de identificar tempranamente cuales son los pacientes que requerirán controles más estrechos u otras alternativas terapéuticas (31-38) entre ellos están: edad menor de 55 años, sexo femenino, raza negra, pobreza, nivel educativo bajo, ama de casa, residente en instituciones geriátricas, mala adherencia, obesidad, uso crónico de un número elevado de medicamentos diferentes a la warfarina, mayor número de hospitalizaciones, cáncer, diabetes, enfermedad renal y hepática crónica, ictus previo, falla cardiaca avanzada, enfermedad pulmonar crónica, hipertiroidismo, hipotiroidismo, epilepsia enfermedades mentales como trastorno bipolar, demencia, depresión mayor, abuso de alcohol y de otras sustancias y polimorfismos en el alelo CYP2C9, VKORC1.

La puntuación SAMeTT2R2 se propuso como una puntuación simple para su uso en la práctica clínica diaria con el fin de ayudar en la toma de decisiones sobre la elección del mejor tratamiento anticoagulante. Fue desarrollada con una mayoría de pacientes caucásicos (20) y la mitad de los participantes tenían puntuación menor a $2(38,40)$. Existen diferencias en las características de cada población que hacen necesario que esta escala se valide en cada una de ellas antes de generalizar su uso $(20,29,39-44)$. En esta muestra el TTR promedio calculado por el método de Rosendaal fue $67.7 \%$ y el PINNR promedio fue $63.6 \%$, que es mayor a lo reportado en otras publicaciones $(29,41)$. El comportamiento del TTR fue un poco diferente respecto al PINRR, el cálculo de calidad en la anticoagulación fue diferente con las dos fórmulas (con el TTR el $60 \%$ de los participantes tenían buena calidad y con el PINNR solo 44.5\%). Chan (45) reportó que un PINRR $\leq 56.1 \%$ es un buen discriminador de TTR $<65 \%$, con sensibilidad de $98.3 \%$, especificidad $50 \%$, valor predictivo positivo $91.9 \%$ y valor predictivo negativo $83.5 \%$. No hay consenso en cuál de las dos técnicas aplicar en la práctica diaria.

Al evaluar la relación entre el SAMeTT2R2 y el TTR/ PINRR no evidenciamos que a mayor puntuación del puntaje, la mediana del TTR/PINNR disminuyera. Se encontraron diferencias significativas al categorizarla cuando se empleó el método PINNR entre el grupo de pacientes con puntaje igual o menor a dos puntos y el grupo de pacientes con puntaje mayor a dos puntos ( $\mathrm{U}$ de Mann-Whitney $=2.22$, $\mathrm{p}=0.02)$. La capacidad discriminativa del SAMeTT2R2 score tuvo áreas bajo la curva cercanas a 0.5 indicando falta de discriminación del SAMeTT2R2 para predecir mal control de la anticoagulación, incluso considerando como punto de corte TTR/PINNR $<36 \%$ que fue el percentil 5 del estudio original (20). En el estudio de Apostolakis (20) la discriminación fue moderada para el percentil 5 del TTR (31 y $36 \%$ ) en cada una de las cohortes de validación interna y externa (c-Index 0.72 (IC 95\% 0.64-0.79) y 0.7 (IC 95\% 0.57-0.82) respectivamente), mas no así con los percentiles 10 y 25 en los cuales fue baja, é0sta ha sido una de las críticas realizadas a este score (22-24). En Uruguay (29), no hubo diferencias en la calidad de la anticoagulación oral con warfarina entre las categorías (favorable y desfavorable) del score SAMe-TT2R2. No hubo diferencia significativa en la media del TTR según la categoría del SAMe-TT2R2 score 
$(53.0 \pm 23.7 \%$ vs $56.2 \pm 20.2 \%, \mathrm{p}=0.447)$. La puntuación del score SAMe-TT2R2 fue similar en los pacientes con TTR $>65 \%$ y $<65 \%$ (1.81 \pm 0.96 vs $1.81 \pm 1.1 ; \mathrm{p}=0.496)$. Tampoco se encontró diferencia entre la calidad de anticoagulación y un puntaje creciente en el score SAMe-TT2R2 $(\mathrm{p}=0.359)$. Otros estudios también han demostrado baja capacidad discriminativa de esta puntuación $(30,43,44)$. Sin embargo estudios con población europea han mostrado utilidad en la aplicación del SAMe-TT2R2 score $(21,28,43,46)$.

Un problema inherente de la puntuación SAMe-TT2R2 es la suposición de que un conjunto fijo de características del paciente predice un curso de por vida de la calidad en el tratamiento con VKA. Esto afecta la validez de constructo y de contenido de la puntuación para su generalización. Cuando un paciente inicia la terapia con warfarina, es difícil predecir en la práctica diaria si será capaz de lograr y mantener un buen control de INRs. Los determinantes del TTR pueden ser multifactoriales y pueden incluir el método, la intensidad del monitoreo, la automonitorización, el soporte social, el hospital y la clínica (especializada frente no especializada) (28). La calidad del control anticoagulante es el resultado de un proceso dinámico con variación individual con relación a la adherencia, estado de salud o enfermedad intercurrente (9). En este estudio se realizó el análisis de cada una de las variables incluidas en el SAMeTT2R2 y ninguna de las variables se relacionó con el riesgo de mala calidad de la anticoagulación con warfarina, similar a lo reportado por Gómez (29), lo cual puede explicar la pobre capacidad de discriminación del score y plantea que hay otras variables que influyen en esta población diferentes a la caucásica. En poblaciones asiáticas variables como edad $<60$ años HR $0.39(0.18-0.84) \mathrm{p}=0.016$ y el ACV/AIT HR $0.64(0.45-0.90) \mathrm{p}=0.01$ (41) tuvieron una relación contraria a la reportada por Apostolakis (20).

Se sabe que un determinante de la calidad de la anticoagulación es la experiencia en el manejo de VKA (9). En la población de este estudio el promedio de TTR fue bueno con respecto a otras poblaciones no caucásicas, todos estaban siendo seguidos en una clínica de anticoagulación; este puede ser un determinante clave para un mejor control y seguimiento $(31,46,47-51)$.

Limitaciones: El estudio fue de diseño retrospectivo y tiene las limitaciones propias de este tipo de estudios. No hay suficientes datos de una cohorte de caucásicos para evaluar su comportamiento en un ambiente latinoamericano, pero será difícil obtener esta información dada la baja prevalencia de esta población en nuestro medio. No tenemos el registro de la información sobre la frecuencia y magnitud de los cambios en la dosis de warfarina, el número de hospitalizaciones que presentaron los pacientes y las complicaciones del tratamiento para hacer su correlación con la puntuación. Tampoco se analizaron otras variables que pueden influir en la calidad de la anticoagulación ni se recopiló datos sobre otras posibles variables de confusión como la distancia de las clínicas de anticoagulación. La aplicabilidad de nuestros resultados a otras poblaciones, con variaciones étnicas, culturales o de tratamiento, es a priori limitada y debe ser abordada en otros estudios.

\section{Conclusión}

La puntuación SAMeTT2R2 en la población estudiada no mostró un buen rendimiento ya que sus variables no se relacionaron con el desenlace TTR $<=65 \%$ y su capacidad de discriminación es baja comparada con el azar por lo cual en esta población no caucásica no se recomienda su aplicación, incluso con puntos de corte diferentes al original. Se considera que los pacientes con FA en anticoagulación asistan a controles en una clínica de anticoagulación con personal entrenado para su seguimiento y en ellas determinar qué características pueden afectar la calidad de la anticoagulación de su población y plantear estrategias para obtener mejores resultados.

\section{Agradecimientos}

A la Fundación Universitaria y EPS Sanitas, por permitirnos realizar la investigación en su programa.

\section{Referencias}

1. January CT, Wann LS, Alpert JS, Calkins H, Cigarroa JE, Cleveland JC Jr, et, al. AHA/ACC/HRS Guideline for the management of patients with atrial fibrillation: a report of the American College of Cardiology/American Heart Association Task Force on Practice Guidelines and the Heart Rhythm Society. J Am Coll Cardiol. 2014; 64: e1-76.

2. Yamanouchi H, Tomonaga M, Shimada H, Matsushita S, Kuramoto K, Toyokura, Y. Nonvalvular atrial fibrillation as a cause of fatal massive cerebral infarction in the elderly. Stroke. 1989; 20: 1653-1656.

3. Hart RG, Pearce LA, Aguilar MI. Meta-analysis: antithrombotic therapy to prevent stroke in patients who have nonvalvular atrial fibrillation. Ann Intern Med. 2007; 146: 857-867.

4. Kirchhof P, Benussi S, Kotecha D, Ahlsson A, Atar D, Casadei B, et, al. European Heart Rhythm Association; European Association for Cardio-Thoracic Surgery: Guidelines for the management of atrial fibrillation: the Task Force for the Management of Atrial Fibrillation of the European Society of Cardiology (ESC). Eur. Heart J. 2016; 37: 2893-2962.

5. Camm AJ, Kirchhof P, Lip GY, Schotten U, Savelieva I, Ernst S, et al. Guidelines for the management of atrial fibrillation: the task force for the management of atrial fibrillation of the European Society of Cardiology (ESC). Eur Heart J. 2010; 31(19): 2369-2429.

6. Skanes AC, Healey JS, Cairns JA, Dorian P, Gillis AM, McMurtry MS, et al. Focused 2012 update of the Canadian Cardiovascular Society atrial fibrillation guidelines: recommendations for stroke prevention and rate/rhythm control. Can J Cardiol. 2012; 28(2): 125-136.

7. National Clinical Guideline Centre (UK) Internet). Atrial fibrillation: The management of atrial fibrillation (CG180). London: National Institute for Health and Care Excellence (UK), 2014 Citado el 19 Jun 2014). Disponible en: http:// guidanceniceorguk/CG180.

8. Ageno W, Gallus AS, Wittkowsky A, Crowther M, Hylek EM, Palareti G. Oral anticoagulant therapy. Antithrombotic therapy and prevention of thrombosis, 9th ed: American College of Chest Physicians evidence-based clinical practice guidelines. Chest. 2012; 141(2): e44S-88S

9. Fauchier L, Poli D, Olshansky B. The SAMe-TT2R2 score and quality of anticoagulation in AF: Can we predict which patient benefits from anticoagulation? Thromb Haemost. 2015; 114: 657-659.

10.Schmitt L, Speckman J, Ansell J. Quality assessment of anticoagulation dose management: comparative evaluation of measures of time-in-therapeutic range. J Thromb Thrombolysis. 2003; 15(3): 213-6.

11. Patel MR, Mahaffey KW, Garg J, Pan G, Singer DE, Hacke W, et al. Rivaroxaban versus warfarin in nonvalvular atrial fibrillation. $N$ Engl J Med. 2011; 365(10): 883-891.

12. Esteve-Pastor MA, Roldán V, Valdés M, Lip GY, Marín F. The SAMe-TT2R2 score and decision-making between a vitamin $\mathrm{K}$ antagonist or a non-vitamin $\mathrm{K}$ 
antagonist oral anticoagulant in patients with atrial fibrillation. Expert Rev Cardiovasc Ther. 2016; 14(2): 177-87.

13. Connolly SJ, Ezekowitz MD, Yusuf S, Eikelboom J, Oldgren J, Parekh A, et al. Dabigatran versus warfarin in patients with atrial fibrillation. $N$ Engl J Med. 2009; 361(12): 1139-1151.

14. Connolly SJ, Pogue J, Eikelboom J, Flaker G, Commerford P, Franzosi MG, et al. Benefit of oral anticoagulant over antiplatelet therapy in atrial fibrillation depends on the quality of international normalized ratio control achieved by centers and countries as measured by time in therapeutic range. Circulation. 2008; 118(20): 2029-2037.

15. Gallego $P$. Common questions in anticoagulation management in atrial fibrillation. Card Electrophysiol Clin. 2014; 6 (1): 79-86.

16. De Caterina RHS, Wallentin L, Andreotti F, Arnesen H, Bachmann F, Baigent C, et, al. Vitamin K antagonists in heart disease: current status and perspectives (Section III). Position paper of the ESC Working Group on Thrombosis--Task Force on Anticoagulants in Heart Disease. Thrombosis and haemostasis. 2013; 110: $1087-1107$.

17. White HD, Gruber M, Feyzi J, Kaatz S, Tse HF, Husted S, et al. Comparison of outcomes among patients randomized to warfarin therapy according to anticoagulant control: results from SPORTIF III and V. Arch Intern Med. 2007; 167: 239-245.

18. Lip GY, Frison L., Halperin JL, Lane CD. Comparative validation of a Novel Risk Score for predicting Bleeding Risk min anticoagulated patients with atrial fibrillation. The HAS-BLED (Hypertension, Abnormal Renal/Liver function, Stroke, Bleeding History or Predisposition, Labile INR, Elderly, Drugs/Alcohol concomitantly) Score. J Am Coll Cardiol. 2011; 57: 173-80.

19. Lau YC, Lip GY. Which drug should we use for stroke prevention in atrial fibrillation? Curr Opin Cardiol. 2014, 29(4): 293-300.

20. Apostolakis S, Sullivan RM, Olshansky B, Lip GY. Factors affecting quality of anticoagulation control among patients with atrial fibrillation on warfarin the SAMe-TT2R2 score. Chest. 2013; 144(5): 1555-1563.

21. Ruiz M, Bertomeu V, Cequier A, Marín F, Anguita M. Validación del score SAME-TT2R2 en una población española de pacientes con fibrilación auricular no valvular tratada con antagonistas de la vitamina k: un subanálisis del estudio califa. Rev Esp Cardiol. 2015; 68 (1): 547.

22. Zhang H, Yang Y, Zhu J. The SAMe-TT 2 R 2 score far from clinical application. Chest. 2014; 145(2): 418-419.

23. Vanerio G. International Normalized Ratio Variability: A measure of anticoagulation quality or a powerful mortality predictor. J Stroke Cerebrovasc Dis. 2015; 24(10): 2223-2228.

24. Skov J, Bladbjerg EM, Bor MV, Gram J. SAMe-TT (2) R (2) does not predict time in therapeutic range of the international normalized ratio in patients attending a high-quality anticoagulation clinic. Chest. 2014; 145(1): 187-188.

25. Holbrook A, Schulman S, Witt DM, Vandvik PO, Fish J, Kovacs MJ, et al. Evidence-based management of anticoagulant therapy: Antithrombotic Therapy and Prevention of Thrombosis, 9th ed: American College of Chest Physicians Evidence-Based Clinical Practice Guidelines. Chest. 2012; 141: e152S - 184S.

26. Rosendaal FR, Cannegieter SC, van der Meer FJ, Briet E. A method to determine the optimal intensity of oral anticoagulant therapy. Thromb Haemost. 1993; 69: 236-9.

27. World Medical Association. Declaration of Helsinki Ethical Principles for Medical Research Involving Human Subjects. JAMA. 2013; 310(20): 2191-2194.

28. Poli D, Antonucci E, Testa S, Hong Lip GY. A prospective validation of the SAME-TT2R2 score: how to identify atrial fibrillation patients who will have good anticoagulation control on warfarin. Intern Emerg Med, 2014; 9 (4): 443-447.

29. Gómez A, Peixoto S, Azcúnaga M, Lluberas N, Silvera G, Álvarez P, et, al. Utilidad del score SAMe-TT2R2 en el control de la anticoagulación oral con warfarina en pacientes con fibrilación auricular no valvular. Rev Urug Cardiol. 2016; 31: 381-389.

30. Lobos-Bejarano JM, Barrios V, Polo-García J, Escobar C, Vargas-Ortega D, Marín-Montañés N, et, al. Evaluation of SAMe-TT2R2 score and other clinical factors influencing the quality of anticoagulation therapy in non-valvular atrial fibrillation: a nationwide study in Spain. Curr Med Res Opin. 2016; 32 (7):1201-7.

31. Baker WL, Cios DA, Sander SD, Coleman CI. Meta-analysis to assess the quality of warfarin control in atrial fibrillation patients in the United States. $J$ Manag Care Pharm. 2009; 15(3): 244-252.
32. Walraven VC, Jennings A, Oake N, Fergusson D, Forster AJ. Effect of study setting on anticoagulation control: a systematic review and metaregression. Chest. 2006; 129(5): 1155-1166.

33. Rose AJ, Hylek EM, Ozonoff A, Ash AS, Reisman JI, Berlowitz DR. Patient characteristics associated with oral anticoagulation control: results of the Veterans AffaiRs Study to Improve Anticoagulation (VARIA). J Thromb Haemost. 2010; 8(10): 2182-2191.

34. Witt DM, Delate T, Clark NP, Martell C, Tran T, Crowther MA, et al. Outcomes and predictors of very stable INR control during chronic anticoagulation therapy. Blood. 2009; 114 (5): 952-956.

35. Witt DM, Delate T, Clark NP, Martell C, Tran T, Crowther MA, et al. Twelvemonth outcomes and predictors of very stable INR control in prevalent warfarin users. J Thromb Haemost. 2010; 8 (4): 744-749.

36. Pokorney SD, Simon DN, Thomas L, Fonarow GC, Kowey PR, Chang P, et al. Patients' time in therapeutic range on warfarin among US patients with atrial fibrillation: Results from ORBIT-AF registry. Am Heart J. 2015; 170(1):141-148.

37. Anguita M, Bertomeu V, Cequier A. Quality of Vitamin K Antagonist Anticoagulation in Spain: Prevalence of Poor Control and Associated Factors. Rev Esp Cardiol. 2015; 68 (9):761-768.

38. Park YK, Lee MJ, Kim JH, Kim SJ, Kim JS, Lee SY, et, al. Lack of association of clinical factors (SAMe-TT2R2) with CYP2C9/VKORC1 genotype and anticoagulation control quality. Journal of Stroke 2015; 17(2): 192-198.

39. Bernaitis N, Keong C, Chen L, Shing J, Chong S, Davey A, et, al. The Sex, Age, Medical History, Treatment, Tobacco Use, Race Risk (SAMe TT2R2) Score Predicts Warfarin Control in a Singaporean Population. J Stroke Cerebrovasc Dis. 2016; 26 (1): 64-69.

40.Solano MH. Adherencia al tratamiento anticoagulante. Hospital de San José. Bogotá dc, Colombia. Repert.med.cir. 2014; 23(3): 189-198.

41. Chan PH, Hai JJ, Chan E, Hua LW, Hung FT, Wong IC, et, al. Use of the SAMe-TT2R2 Score to Predict Good Anticoagulation Control with Warfarin in Chinese Patients with Atrial Fibrillation: Relationship to Ischemic Stroke Incidence. PLoS One. 2016; 11(3): e 0150674.

42. Barrios V, Escobar C, Prieto L, Osorio G, Polo J, Lobos JM, et al. Control de la anticoagulación en pacientes con fibrilación auricular no valvular asistidos en atención primaria en España. Estudio PAULA. Rev Esp Cardiol. 2015; 68: 769-76.

43. Abumuaileq RRY, Abu-Assi E, Raposeiras RS, López A, Redondo AD, Álvarez DI. Evaluation of SAMe-TT2R2 risk score for predicting the quality of anticoagulation control in a real-world cohort of patients with non-valvular atrial fibrillation on vitamin-K antagonists. Europace. 2015; 17: 711-717.

44. Ruiz-Ortiz M, Bertomeu V, Cequier Á, Marín F, Anguita M. Validation of the SAMe-TT2R2 score in a nationwide population of nonvalvular atrial fibrillation patients on vitamin K antagonists. Thromb Haemost. 2015; 114: 695-701.

45. Chan PH, Li WH, Hai JJ, Chan EW, Wong IC, Tse HF, et, al. Time in Therapeutic Range and Percentage of INRs in Therapeutic Range as measure of quality of anticoagulation control in atrial fibrillation patients. Canadian Journal of Cardiology. 2015; 31 (11): 1-23.

46. Marín F, Gallego P, Gálvez J, Jover E, Vicente V, Lip GY. Utilidad de la escala SAMe-TT2R2 en la predicción de la calidad de la anticoagulación y eventos adversos en pacientes con fibrilación auricular no valvular bajo tratamiento anticoagulante. Rev Esp Cardiol. 2014; 67 (1): 240.

47. Taboada LB, Silva LE, Montenegro AC. Beneficios de la clínica de anticoagulación. Acta Med Colomb. 2013; 38: 239-243.

48. Bloomfield HE, Taylor BC, Krause A, Reddy P, Greer N, MacDonald R, Rutks I, Wilt TJ. Safe and Effective Anticoagulation in the Outpatient Setting: A Systematic Review of the Evidence. VA Evidence-based Synthesis Program (ESP) Center, Center for Chronic Disease Outcomes Research, Minneapolis VA Medical Center. 2011.

49. Wilson SJ, Wells PS, Kovacs MJ, Lewis GM, Martin J, Burton E, et al. Comparing the quality of oral anticoagulant management by anticoagulation clinics and by family physicians: a randomized controlled trial. CMAJ 2003; 169(4): 293-8.

50. Macik BG. The future of anticoagulation clinics. J Thromb Thrombolysis 2003; 16(1-2): 55-9.

51. Van Walraven C, Jennings A, Oake N, Fergusson D, Forster AJ. Effect of study setting on anticoagulation control: a systematic review and metaregression. Chest 2006; 129(5): 1155-66. 